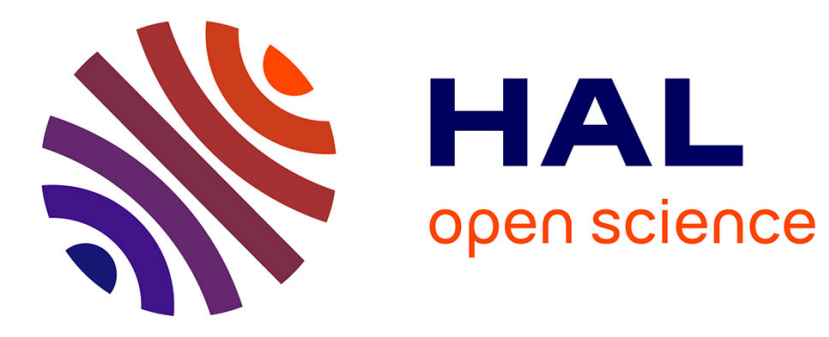

\title{
WSN-inspired Sleep Protocols for Heterogeneous LTE Networks
}

\author{
Iulia Tunaru, Hervé Rivano, Fabrice Valois
}

\section{To cite this version:}

Iulia Tunaru, Hervé Rivano, Fabrice Valois. WSN-inspired Sleep Protocols for Heterogeneous LTE Networks. PE-WASUN - 10th ACM International Symposium on Performance Evaluation of Wireless Ad Hoc, Sensor, and Ubiquitous Networks, Nov 2013, Barcelona, Spain. pp.1-8, 10.1145/2507248.2507267. hal-00867937

\section{HAL Id: hal-00867937 \\ https://hal.inria.fr/hal-00867937}

Submitted on 30 Sep 2013

HAL is a multi-disciplinary open access archive for the deposit and dissemination of scientific research documents, whether they are published or not. The documents may come from teaching and research institutions in France or abroad, or from public or private research centers.
L'archive ouverte pluridisciplinaire HAL, est destinée au dépôt et à la diffusion de documents scientifiques de niveau recherche, publiés ou non, émanant des établissements d'enseignement et de recherche français ou étrangers, des laboratoires publics ou privés. 


\section{WSN-inspired Sleep Protocols for Heterogeneous LTE Networks}

\author{
Iulia Tunaru \\ CEA \\ CEA-Leti, Minatec Campus \\ 38054 Grenoble, France \\ iulia.tunaru@cea.fr
}

\author{
Hervé Rivano \\ Inria, Université de Lyon \\ INSA-Lyon, CITI-Inria \\ F-69621 Lyon, France \\ herve.rivano@inria.fr
}

\author{
Fabrice Valois \\ Université de Lyon, Inria \\ INSA-Lyon, CITI-Inria \\ F-69621 Lyon, France \\ fabrice.valois@inria.fr
}

\begin{abstract}
The tremendous increase of the traffic demand in cellular networks imposes a massive densification of the traditional cellular infrastructure. The network architecture becomes heterogenous, in particular 4G networks where LTE micro eNodeBs are deployed to strengthen the coverage of macro eNodeBs.

This densification yields major issues related to the energy consumption of the infrastructure. Indeed, there is fixed and significant amount of energy required to run each additional node, whatever the traffic load of the network. Mitigating this fixed energy consumption is therefore a major challenge from a societal and economical viewpoint.

Extensive researches about energy-saving highlight that to save energy the better strategy is to switch off the radio part of nodes. This is the heart of wireless sensor networks energy-saving strategies, even though the objective for WSN is to maximize the battery life of each individual nodes.

In this paper, we develop a parallel between the principles of WSN protocols and the requirements of cellular infrastructures. We then propose a distributed and localized algorithm to dynamically switch off and on the micro eNodeBs of an LTE heterogeneous network following the traffic demand evolution in time and analyze it in terms of energy savings. We show that one can expect energy savings of approximately $12 \%$ when implementing sleep modes whereas the energy cost for sending the traffic decreases by $24 \%$.
\end{abstract}

\section{Categories and Subject Descriptors}

C.2.1 [Network Architecture and Design]: Wireless communication

\section{Keywords}

Energy optimization; switch off mechanisms; cellular network; topology control

Permission to make digital or hard copies of all or part of this work for personal or classroom use is granted without fee provided that copies are not made or distributed for profit or commercial advantage and that copies bear this notice and the full citation on the first page. Copyrights for components of this work owned by others than ACM must be honored. Abstracting with credit is permitted. To copy otherwise, or republish, to post on servers or to redistribute to lists, requires prior specific permission and/or a fee. Request permissions from permissions@ acm.org.

PE-WASUN'13, November 04 - 08 2013, Barcelona, Spain (c) 2013 Association for Computing Machinery. ACM acknowledges that this contribution was authored or coauthored by an employee, contractor or affiliate of the national government. As such, the Government retains a nonexclusive, royalty-free right to publish or reproduce this article, or to allow others to do so, for Government purposes only.

Copyright 2013 ACM 978-1-4503-2360-4/13/11 ..\$15.00

http://dx.doi.org/10.1145/2507248.2507267.

\section{INTRODUCTION}

The number of mobile phone subscribers approaches 6 billion [1]. Wireless data is the fastest growing traffic in the Internet and the density of required throughput is forecasted to narrow a Gigabit/s per square kilometer where up-todate $4 \mathrm{G}$ deployments provides about $100 \mathrm{Mb} . \mathrm{s}^{-1} / \mathrm{km}^{2}[2]$. Closing this gap requires a massive densification of the cellular infrastructure. In particular, this means a generalization of an heterogeneous architecture in which traditional macrocells, eNodeBs in the context of $4 \mathrm{G}$ LTE networks, are strengthen by the deployment of microcells, depicted in Fig. 1. The new concept of LTE relay nodes implies the existence of two radio interfaces (one for the users and another one for the relay backhaul). The terminals in this region have then the opportunity to attach to one of the microcell which relay the traffic to the macro. Together with inter-cell interference mitigation cooperative algorithms, microcells provide very high quality of signal to users using less resources [3].

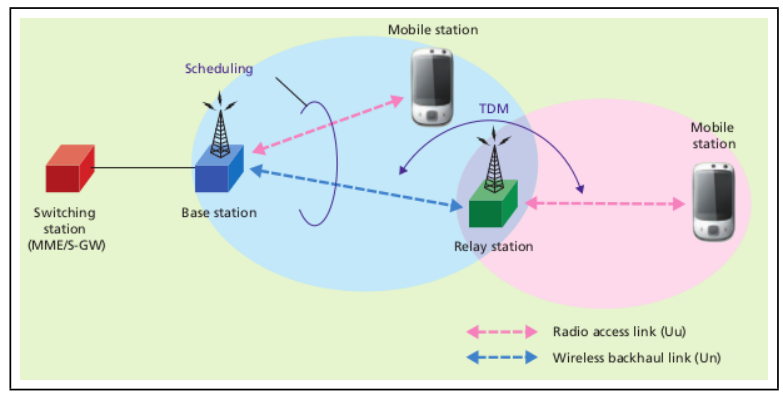

Figure 1: LTE relay network architecture

Envisioning such a densification of the infrastructure yields tremendous issues considering the energy consumption counterpart.This is an economic issue with the rise of the financial cost of energy when $60 \%$ to $80 \%$ of the total energy expenditure of a telecommunication operator is already concentrated in the Radio Access Network (RAN) [4]. This is also a legitimate environmental concern since the consumption of nowadays nation-wide RANs is a few percent of the total energy consumption of industrialized countries, and is forecast to exceed the production of nuclear reactor [1].

Consequently, the GreenTouch initiative have been set up gathering many industrial and academic actors of the telecommunication sector for targeting a decrease of several orders of the energy consumption of network infrastructures [5]. Similarly, the European project EARTH aims at finding solutions for decreasing energy consumption of the access 
network and as part of its objectives [6]. An energy efficiency evaluation framework $\left(\mathrm{E}^{3} \mathrm{~F}\right)$ for 3GPP LTE infrastructure has been proposed $[1,7]$. The $\mathrm{E}^{3} \mathrm{~F}$ proposes a detailed power model for base stations (eNodeBs), long-term traffic models that describe the fluctuations over a day, and large scale deployment models for large geographical areas. The numerical simulations of this paper are based on this model. One of the key findings of the EARTH analysis is that, on average, a vast amount of the resources are idle. Measurements on a $3 \mathrm{G}$ network in a major European city assess this [1].

Many papers have focused on energy saving approaches dealing with power control, leveraging both advanced technologies (e.g. advanced antenna design, beamforming, cooperative communications) and energy efficient network planning and deployments. In particular, an optimized deployment of micro base stations can jointly minimize the power consumption per surface and maximize the throughput [8]. Other have shown that the joint optimization of resource allocation, routing and power control allows to achieve significant gains in terms of throughput and mean transmit power $[9,10]$. Unfortunately, at low loads, the energy efficiency of contemporary RF systems is particularly poor. In other terms, they are said to be little load proportional which means that optimal power control policies is not enough to mitigate the fixed power consumption of the system. An obvious direction is to modify the electronic architecture of the equipments in order to achieve higher efficiency at lower loads. On the other hand, some energy costs are unavoidable unless some functionalities are literally switched off, including the radio communication part. A major share of the energy savings comes therefore from the network architecture and management [11]. In this paper, we assume that the eNodeBs have a sleeping mode which switch off all energy intensive functionalities, keeping up a signaling interface. We the focus on algorithms which improve the network energy efficiency by concentrating the traffic on some nodes, so that other could then enter a sleep mode.

Sleeping mode for radio access networks seems particularly interesting for several reasons. First, access cellular networks show a certain level of redundancy which is needed to absorb the increasing traffic demand and the highly asymmetric daily traffic profile, especially for data. Secondly, the traffic does not only vary between peak and off-peak hours but also geographically, which means that self-organizing solutions can leverage also small variations of the traffic at peak hours. Finally, leveraging sleep modes via a signaling protocol among eNodeBs is a flexible solution which is independent and complementary to enhancements of the electronic component and well defined network designs.

On the one hand, several recent publications deal with the implementation of sleep protocols in hierarchical cellular networks, considering heterogeneous macro-micro deployments $[4,12,13]$, or with general sleep modeling using random graphs $[14,15]$. Most of these works consider complete switch off of the radio equipment. This strategy is more efficient when the equipments are little load-proportional and when networks are highly connected [14]. Using a simple model, the fraction of base stations that could be switched off for different cell layouts in order to save energy and respond to an overall traffic capacity demand can be calculated
[4]. This study takes into account the load proportionality of equipments and proposes a centralized implementation. A stochastic analysis on random deployments also assess the relevance of these techniques when the objective is to guaranty a given outage probability [15]. Similar techniques have been proposed on wired core networks [16], where some network interfaces that are not used in the most of the data traffic forwarding paths are switched-off. This is done by keeping the connectivity above a given threshold on the one side with the aim of saving the network interface energy on the other side.

On the other hand, sleep protocols and duty cycle algorithms have been the subject of extensive research in the field of wireless sensor networks. WSN have recently become popular due to the rapid progress in the field of MEMS technologies and wireless communications which contributed to the development of inexpensive wireless nodes. Such networks (Fig. 2) are mainly characterized by high density, random repartition of nodes capable of auto-organization, mesh topology, low traffic and low energy consumption. Energy efficiency is a very important aspect in WSN because it conditions the lifetime of the network. We can hence question ourselves whether and which algorithms from the WSN domain could be suitable to cellular networks and to what extent, assuming that we consider eNodeBs as equivalents to wireless sensors (both routing and non-routing nodes).

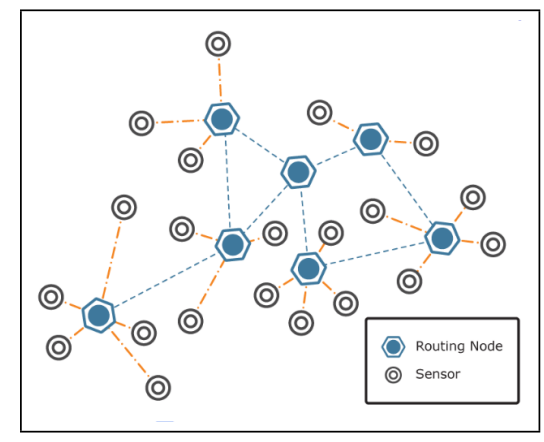

Figure 2: Classic WSN topology

It can be noticed that LTE shifts cellular philosophy towards a certain degree of decentralization with the decision of concentrating certain $3 \mathrm{G}$ RNC-specific radio function in the eNodeBs. For cooperation purposes among nodes, a low power signaling interface, the X2 interface, is implemented in the nodes. This allows for implementing distributed algorithms among eNodeBs if we assume that a eNodeB can be awaken through the X2 interface.

These remarks and the need of implementing efficient sleep modes foster the orientation of this work toward the study of WSN-inspired sleep protocols for LTE networks starting from the scenario of a classical heterogeneous cellular network. The organization of this paper is as follows. The problem statement is discussed in Section 2. An in-depth description of our distributed and localized propositions are presented in Section 3. The network assumptions and the performance results are discussed in Section 4. Conclusions regarding our propositions will be drawn in the last section. 


\section{PROBLEM STATEMENT}

This work deals with the optimization of the energy consumption of LTE base stations or relay nodes by using sleep modes. The algorithms and protocols that will be put forward are inspired from Wireless Sensor Networks (WSN) sleep algorithms which deal with coverage and connectivity criteria [17, 18]. This means that in WSN, which are dense networks, sensors might be put to sleep after it is verified that every point in their surveillance area is covered by at least one other sensor that remains awake and that all the sensors are connected to a gateway in order to be able to forward their information.

Another way to achieve energy efficiency in WSN is the use of topology control algorithms [19]. While sleep scheduling algorithms are closely related to the evaluation of coverage and connectivity of the network, topology control for energy savings can be done via the adjustment of the transmission power of each sensor with the constraint of keeping the network connected at a certain degree.

This parallel between cellular networks and WSN is possible mainly because of the coverage redundancy present in heterogeneous cellular networks and the development of LTE-Relay, whose standardization is still in course. In order to judge the feasibility of this convergent approach, several points should be considered carefully:

- the strategies of WSN algorithms dealing with coverage and connectivity when sleep modes and topology control are used;

- the energy model used in WSN;

- the description of the energy efficiency of LTE eNodeBs (energy model, parameters etc.);

- the context differences and the adaptations that need to be done in order to apply WSN algorithms to LTE networks;

- the performance evaluation.

Moreover, it is important to highlight:

- The sensing model of WSN does not take into account any resource constraint, in other terms, coverage studies do not include, in their calculations, the capacity parameter. This is very important in cellular networks as it is the main focus of radio access networks which are designed with regard to a user density constraint.

- The difference between the energy models of WSN and LTE eNodeB. The energy expenditure of a node can be considered constant while the node is on and null when the node is off. LTE eNodeB have a high fixed operating cost added to a cost proportional to their emitting power level as depicted in Fig. 3.

- In cellular networks, total energy consumption is more important than low communication overhead or lifetime of the network which are crucial in the case of WSN. Also, we can assume that messages between eNodeBs use a reliable transmission thanks to the RRC layer, which is not the case in WSN where algorithms are strongly influenced by message losses.
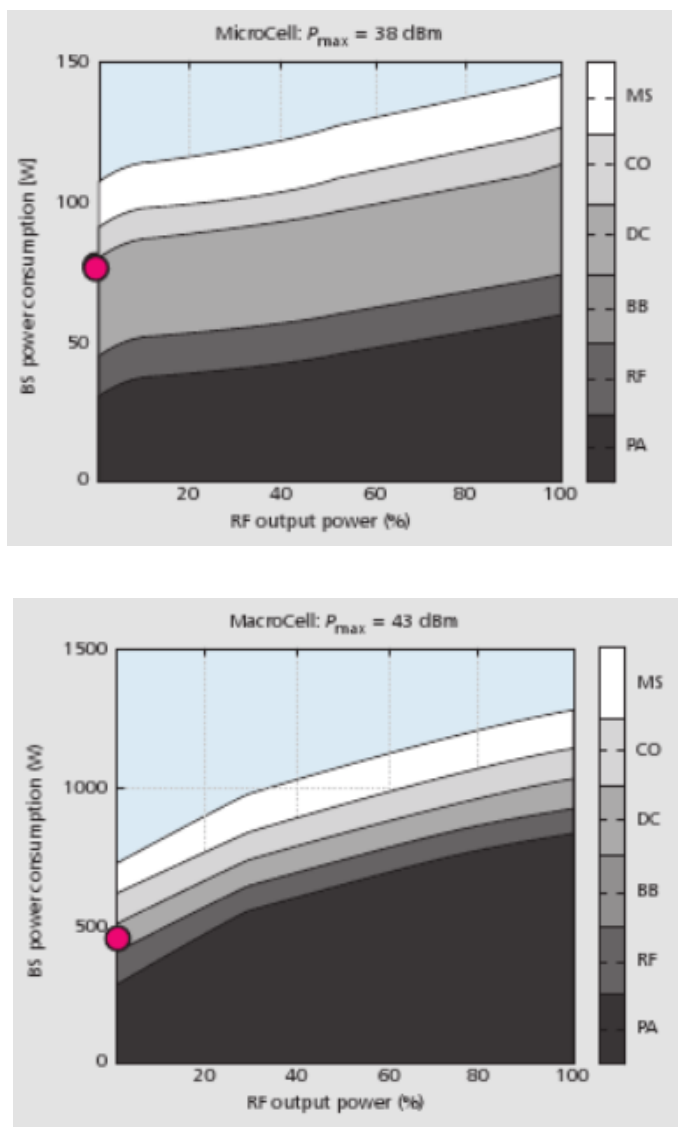

Figure 3: Power consumption vs load : 10MHz LTE, 2x2 MIMO. Micro and 3 sectors Macro eNodeB [7]

\section{DISTRIBUTED AND LOCALIZED ALGO- RITHMS FOR ENERGY-SAVING IN LTE}

In this work, we study two different approaches for switching off eNodeBs. Both are designed to take as input parameter the downlink data traffic repartition over $24 \mathrm{~h}$ which will be presented in Section 4.1. For now, it is sufficient to keep in mind that there are large differences in the traffic demand between day and night, which will be the basis of the start-up of the sleep procedure. The presented solutions consist of slightly different communication protocols, both inspired from the WSN literature.

In the general case, without any algorithm, all the eNodeBs work independently and consume an amount of energy corresponding to their own traffic pattern and coverage areas. When applying a sleep algorithm, there is coordination between base stations, so that some of them could switch off. So, after a switch off, eNodeBs that stay awake recalibrate their traffic demand and are expected to consume slightly more. Nonetheless, overall network energy gains are expected from the important load-independent costs that will be saved by switching off some eNodeBs.

\subsection{Network architecture requirements}

We consider an heterogeneous LTE cellular network as those deployed in urban area $[11,9]$. The heterogeneity of these networks comes from the various scales of access points. Classical macro eNodeBs are strengthen by micro eNodeBs in 
streets, indoor femtocells, etc for capacity and coverage issues. In this work, we focus on outdoor macro with several micros. We assume that any two eNodeBs with overlapping coverage are connected through a X2 interface. This is in particular the case for any micro eNodeBs with their macro eNodeB. Basically, X2 interface is used for control traffic as mobility management, connectivity issues or traffic monitoring. In our work, we assume that the X2 interface is low power, and can awake an eNodeB in sleep mode. We then propose to extend them to support the traffic dedicated to the sleep mode management protocol presented in the following. Beyond the actual messages of the protocols we propose, the dynamics of the topology induced by the switching of the cells will increase the usual X2 traffic. Minimizing the volume of traffic required and induces by our protocols is a challenge that will be addressed in a future work.

\subsection{Distributed sponsor-based switch off strat- egy}

At first sight, a relevant strategy is a sponsor-based approach in which every eNodeB monitors its average traffic load and, once this measurement drops below a given threshold, asks its neighbors for permission to sleep [?]. On receiving such a request, each eNodeB calculates its available "sponsoring" capacities, that is how much of the load of the requesting eNodeB it is able to take on. If the neighbor considers that it is capable of "sponsoring" the eNodeB, it acknowledges it. After receiving all the answers, the eNodeB can estimate if its load can be managed by its neighboring cells. If not, it cancels the procedure, otherwise it forces the handovers of its clients to its sponsoring neighbors and switches off after that. A eNodeB remains asleep until the traffic increases and one of its neighbors asks for help.

This naïve solution presents some drawbacks. First of all, even though it tries to take a decentralized and localized approach, the decision making process is shifted from the eNodeB actually wanting to switch off to its neighbors. The fact that the neighbors are entirely responsible of the verifications adds complexity to the resource verifications. Secondly, this shift can lead to inconsistencies or inefficiencies in the decision process. Wrong decisions could arise if the actual load of the eNodeB changes while its neighbors do the computations. Finally, as the permission to sleep is totally uncorrelated to the node doing the traffic measurements, the way to react and adapt the behavior of a eNodeB after a sleep rejection is not obvious. All these observations plead for a more localized decision process, which is the guideline of the communication protocol presented next.

\subsection{Local rating-based switch off strategy}

In order to cope with the aforesaid issues, the main idea is to localize the decision making of the sleep procedure. The start-up is based on the average load computation done periodically, after a certain delay. The delay computation for the average traffic load is inversely proportional to a local rating, which encodes the priority for a eNodeB to go to sleep. This parameter is initialized by the network operator, evolves during the day, as described in the following, and is reset every $24 \mathrm{~h}$ or after a sleep period. For example, the macro eNodeB would have a null rating since it is not allowed to switch off, while the micro eNodeBs would have a large rating if they are situated in an area where the traffic variations are

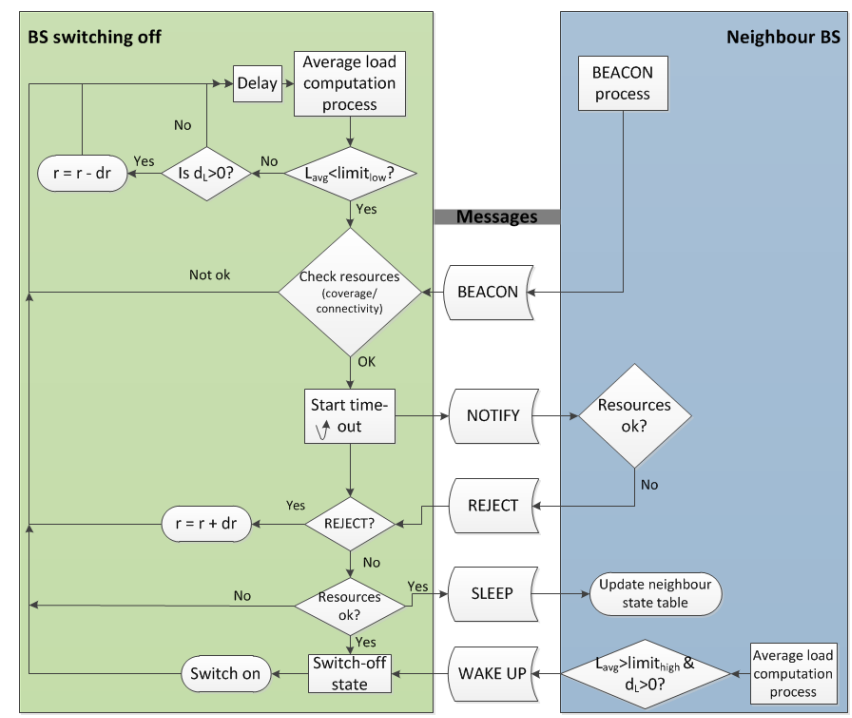

Figure 4: Switch off protocol diagram

well known, relatively abrupt and the traffic is particularly low for long periods of time (e.g. office buildings).

A diagram of the protocol is presented in Fig. 4 while the definitions of the main parameters can be found in table 1 . (a)
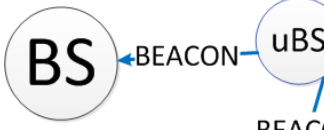

BEACON

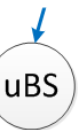

(b)
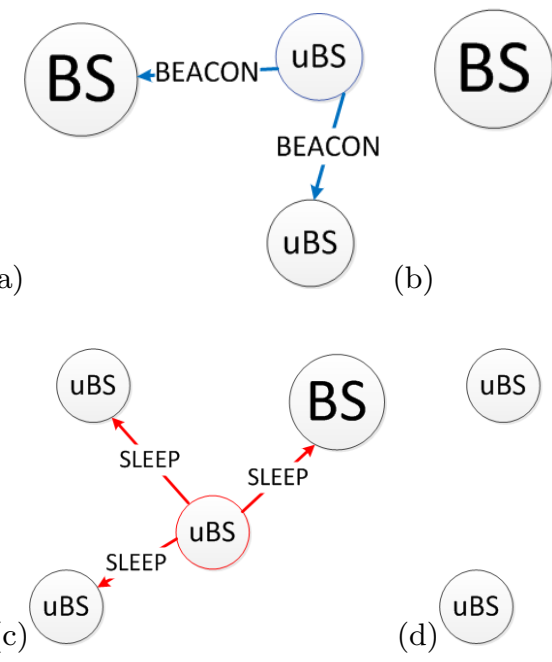

(d)

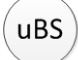

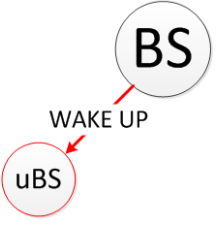

Figure 5: Message exchanges for switch off decision: (a) BEACON , (b) REJECT , (c) SLEEP , (d) WAKE UP

Nodes periodically broadcast BEACON messages indicating position, capacity and traffic load (Fig. 5-a). The time between two beacons $\left(T_{b}\right)$ should be shorter than the load computation delay $\left(d_{r}\right)$. This delay is computed as $d_{r}=$ $T_{\max } / r+b c k$ where $T_{\max }$ is an algorithm parameter representing the maximum delay for a base station, $r$ is the rating and $b c k$ is a random backoff for further desynchronization. After $d_{r}$, every eNodeB computes its average load $\left(L_{a v g}\right)$ and the derivative of the average load $\left(d_{l}\right)$. If $L_{a v g}$ exceeds a given threshold limit $_{l o w}$ and if $d_{l}>0$, it decreases its rating to avoid too frequent evaluations. If $L_{\text {avg }}<$ limit $t_{\text {low }}$, it initiates the sleep procedure, during which it would reject 


\begin{tabular}{|c|l|}
\hline Parameter & Definition \\
\hline$T_{t e}$ & Dispatcher's traffic setting period \\
\hline$T_{b}$ & Beaconing period \\
\hline$d_{r}$ & Load computation delay \\
\hline$t_{\text {out }}$ & time-out delay \\
\hline limit $_{\text {low }}$ & Lower threshold (sleep) \\
\hline limit $_{\text {high }}$ & Higher threshold (wake-up) \\
\hline$p S e c$ & $\begin{array}{l}\text { \% of resources of a cell available } \\
\text { for neighboring eNodeBs to sleep }\end{array}$ \\
\hline
\end{tabular}

Table 1: Parameter definition

all other sleep initiatives from its neighbors. Otherwise, it just loops.

Based on the informations from the BEACON messages, the node evaluates if it can transfer its remaining load to its neighbors, starting with the micro eNodeB and checking the macro eNodeB lastly. If the eNodeB finds a feasible distribution of its load among its neighbors, it sends NOTIFY messages to the concerned neighbors indicating the supplementary load for each one and a time-out delay $\left(t_{\text {out }}\right)$ that it is willing to wait before actually switching off. If it is answered a REJECT (Fig. 5-b), it increases its rating and waits for $d_{r}$ before re-evaluating its average load. If no answer is received, it checks again the evolution of its load during the time-out to avoid inconsistencies, broadcasts a SLEEP message and finally enters sleep mode (Fig. 5-c). It can be switched on again by a WAKE UP message from one of its neighbors on the X2 interface (Fig. 5-d).

At the reception side, a node receiving NOTIFY verifies whether it is actually capable of recovering the mentioned load without saturating. If not, it sends a REJECT before the time-out expires. When receiving a SLEEP message, it updates its neighbors' state table. In the meantime, each node also checks if its average load exceeds another threshold $\left(\right.$ limit $\left._{\text {high }}\right)$ and if $d_{l}$ is positive. In this case, if there are eNodeB neighbors which are asleep, a WAKE UP message is sent to one of them.

In comparison to the sponsor-based solution, the ratingbased algorith is simpler and more stable because the node that wants to switch off controls entirely its state transitions, while its neighbors will only react. We can therefore talk about a greedy localized algorithm. Even though this algorithm does not guarantee $100 \%$ consistent decisions because of the possible evolutions of the traffic loads during periods of time-out, solutions to avoid degradations have been provided: supplementary load verifications before sending sleep messages, tests on the derivatives in order to detect load trends, parametrizable security percentages for load verifications in order to avoid saturation.

\section{PERFORMANCE EVALUATION}

In this section, we will go over the simulations done on a heterogeneous network scenario, and over the results obtained with the numerical parameters presented in table 2 .

\subsection{Network model and scenario}

We consider an heterogeneous urban scenario based on the following pattern. A central macrocell LTE eNodeB covers approximately $500 \mathrm{~m}$ and is strengthen by 30 micro eNodeB, each covering $50 \mathrm{~m}$. As described in Section 3.1, overlapping micro eNobeBs are connected through their X2 interfaces for

\begin{tabular}{|c|c||c|c|}
\hline Parameter & Value & Parameter & Value \\
\hline$T_{b}$ & 1 minute & $T_{\text {te }}$ & 1 minute \\
\hline max $d_{r}$ & $1 \mathrm{~h}$ & $t_{\text {out }}$ & $10 \mathrm{~s}$ \\
\hline \hline \multicolumn{2}{|c||}{ Parameter } & Micro cells & Macro cells \\
\hline \multicolumn{2}{|c|}{ limit $_{\text {low }}$} & Capa $\times 1 / 4$ & 0 \\
\hline \multicolumn{2}{|c|}{ limit $_{\text {high }}$} & Capa $\times 3 / 4$ & Capa $\times 7 / 8$ \\
\hline \multicolumn{2}{|c|}{$p e c$} & 0.75 & 0.25 \\
\hline
\end{tabular}

Table 2: Parameters used for the set of simulations

signaling purposes. Our algorithm leverages this connectivity. An example of generated network topology is depicted in Fig. 6. The edges between eNodeBs represent X2 interfaces connectivity.

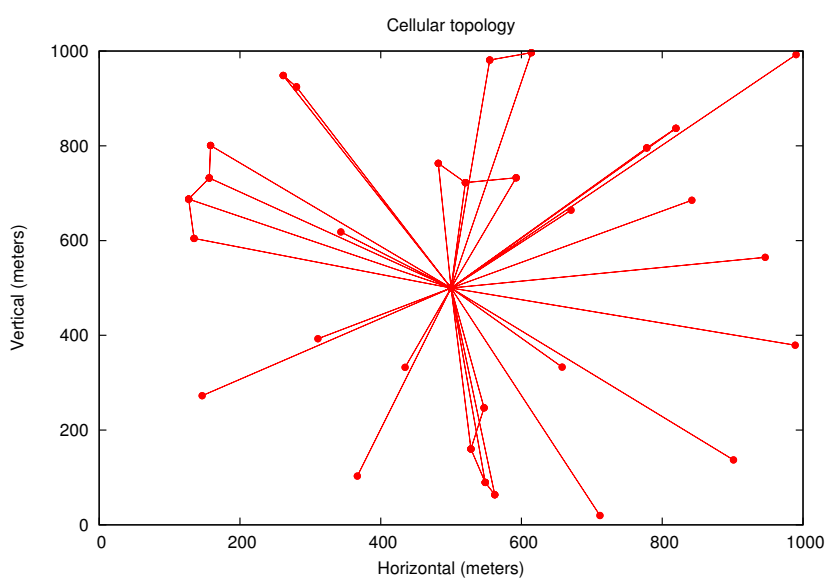

Figure 6: Example of generated network topology

The resource allocation is done using a $3 \times 10 \mathrm{MHz}$ LTE bandwidth which provides $150 \mathrm{PRB}$ (for "Physical Resource Block", the unitary capacity of LTE): $80 \%$ of the resources are allocated to the macro eNodeB and $20 \%$ with frequency reuse 1 to the micro eNodeBs. The energy consumption model of eNodeBs is the one from [7], described in Section 2.

We model the traffic variation of users during the day. The traffic profile described in the EARTH Project [1] is used to generate an approximative sinusoidal traffic repartition for all the eNodeBs, and depicted in Fig. 7. This pattern is most probably characteristic to a residential zone because the peak is around 9pm but we are only interested in the aspect of the long-term variations, which we assume to be the same in the case of other urban areas.

All results are computed with a confidence interval of $95 \%$. In order to assess the energy gains and the capacityenergy trade-off, we define the following metrics: power consumption of each base station, total energy consumption, network capacity and energy cost of a PRB. We evaluate our algorithm by comparing it to the scenario where nodes cannot switch off.

\subsection{Base station level metrics and energy gains}

The effect of the sleep algorithm on the load of the base stations is illustrated in Fig. 8 which shows the load attributed to each micro eNodeB. The peaks that can be noticed for 

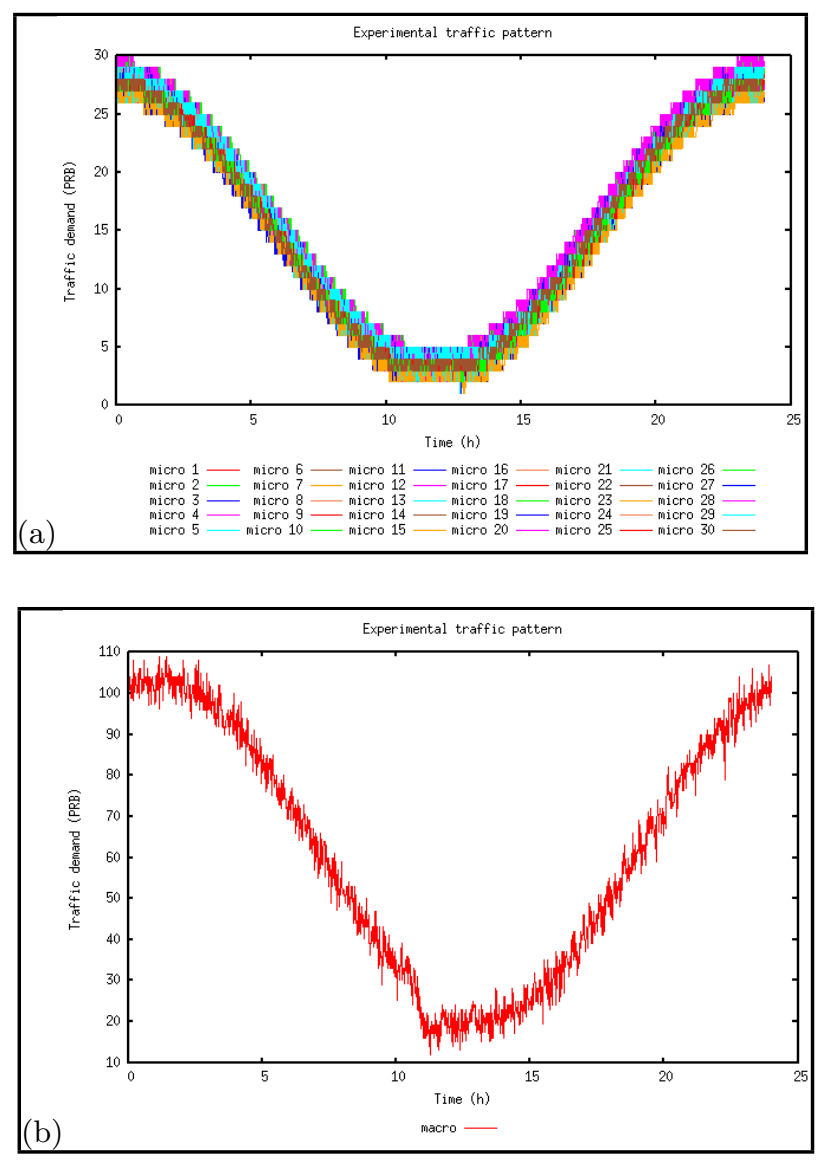

Figure 7: Combined microcells traffic (a) and Macrocell traffic (b) patterns

some of the eNodeBs represent the instant load received from a neighbour that has just switched off. After a while, the load is redistributed uniformly between the neighbours of the switched off eNodeB and the peaks decrease. It can also be noticed that there is no particular order for the wake-up transitions of the eNodeBs.

The actual impact of the algorithm can also be noticed in the power profiles of two micro base stations: one that switches off (Fig. 9-a) and one that does not turn off and absorbs the traffic of its neighbor (Fig. 9-b). With the actual set of parameters, the number of transitions for a eNodeB during $24 \mathrm{~h}$ is usually one because the thresholds are fairly strict in order to avoid monopolising of the macro resources.

The energy consumption of the whole system depends on the number of eNodeBs that switch off, which is slightly dependent on the network topology. For a network with an average node degree of 2 , a gain of $12,3 \%$ is reachable.

\subsection{Network level metrics}

When applying the sleep algorithm, the network capacity, which is the sum of the capacities of all the base stations, will obviously decrease. The simulation in figure 8 shows the evolution of the network capacity, as expected because of the switch off and the switch-on of certain eNodeBs. Without switch-off algorithms, the capacity would remain fixed at 1020 PRB. Fig. 8 shows that the available capacity is always greater than the load. Moreover, the capacity plot is shifted
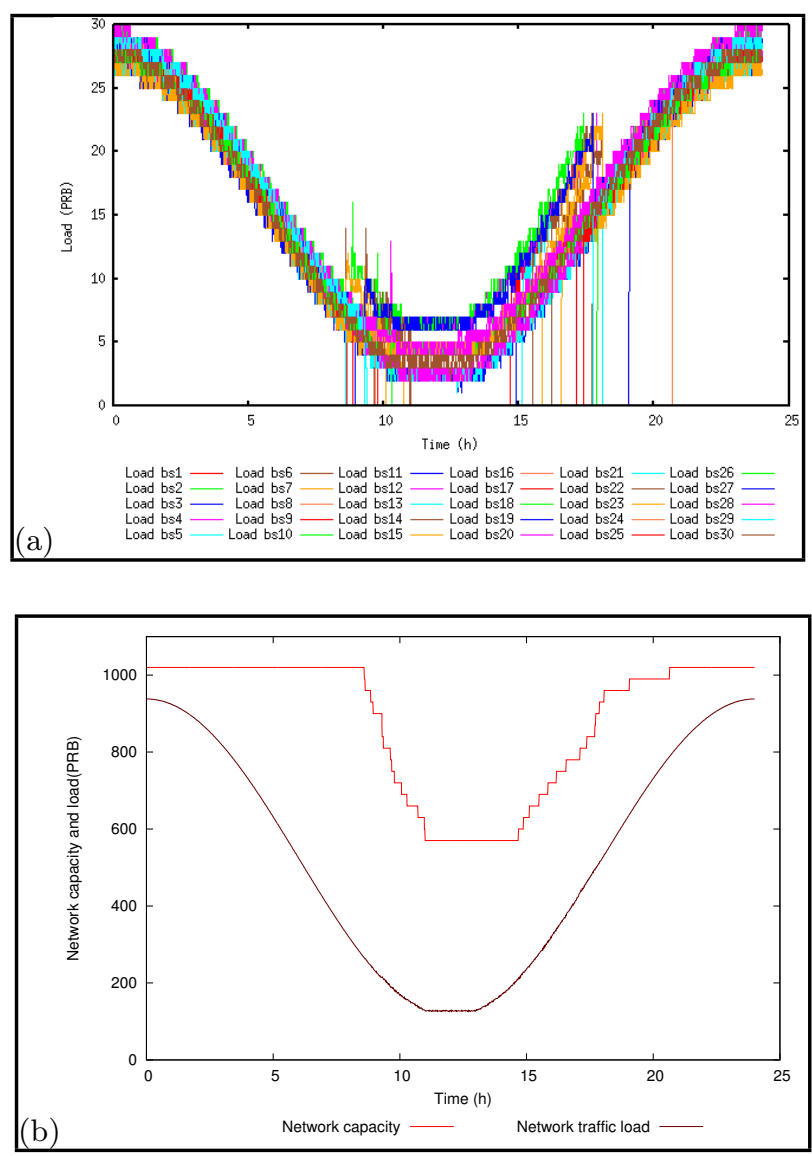

Figure 8: Per cell load (a) and Network capacity and load (b)

on the right compared to the load plot. It mean that the switch-on of cells (when the capacity increases) are done when it is really needed, letting eNodeBs in switch-off mode as long as possible. Nevertheless, one can note that the capacity keeps very high when there is a significant drop in the network traffic load. This is mainly due to the alwayson macro eNodeB and because our choice of parameters is very conservative in order to avoid inconsistent decisions. We estimate that higher levels of adaptation are possible if we loosen the different resource verification constraints (e.g. with larger low and high thresholds).

\begin{tabular}{|l|c|c|}
\hline Metric & Normal mode & Sleep mode \\
\hline Mean energy / PRB capa (EOC) & 0.072 & 0.074 \\
\hline Mean energy / PRB load (EOL) & 0.219 & 0.167 \\
\hline
\end{tabular}

Table 3: Average cost of a PRB over 30 simulations

The last computed metrics are the ratios energy consumption/capacity (denoted EOC) and energy consumption/load $(E O L)$ for the whole network. Every time the load changes, we compute $E O C=\frac{\Sigma E_{i}}{\Sigma C_{i}}$ and $E O L=\frac{\Sigma E_{i}}{\Sigma L_{i}}$ over the last time interval. $E_{i}$ represents the energy consumption of $B S_{i}$ over the last time interval and $C_{i}$ and $L_{i}$ are the capacity and the traffic load, respectively, of switched on base stations. Using this kind of results, which are shown in Fig. 10-a and Fig. 10-b, the average energy cost of a PRB can be computed 

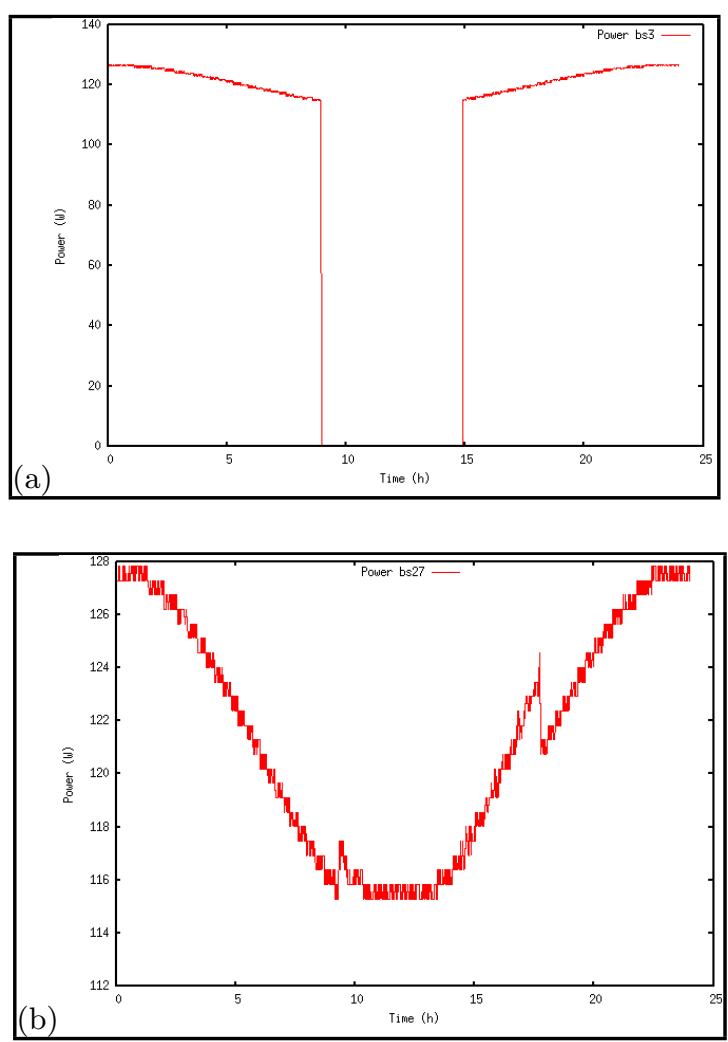

Figure 9: Power consumption of switched off eNodeB (a) and absorbing traffic one (b)

by averaging the above metrics over $24 \mathrm{~h}$. After running 30 simulations without applying any algorithm and another 30 with our sleep algorithm, and choosing a confidence interval of $95 \%$ for the average energy cost of a PRB, we obtain the results illustrated in Table 3 . One can see that the cost of a unit of capacity increases with sleep algorithms. Indeed, there when switching off some eNodeBs, the capacity drops. The energy drops too, but slower. On the other hand the cost of a unit of load decreases, which is what is actually paid. The results show that the energy cost per offered PRB $(E O C)$ increases by $2.2 \%$ while there is a significant saving of $23.9 \%$ in the energy cost of a consumed PRB (EOL).

\section{CONCLUSION}

In this work, we investigate the possibility of adapting WSN sleep strategies to heterogeneous cellular networks. The algorithms and protocols design takes care of several key aspects of the parallel between WSN and cellular networks.

First, there is a certain philosophy difference between the distributed, localized approaches of WSN and cellular networks because the latter are largely hierarchical. In particular, the emergence of the X2 interface, which allows low power eNodeB to eNodeB signaling communications, and the rise of LTE contribute to shifting cellular philosophy towards cooperation and offers the possibility of implementing localized self-optimization techniques.

Secondly, the capacity constraint of cellular networks is one of the strongest considerations that impact the adaptation of WSN algorithms. It is in fact a constraint for switch off procedures which adds to the ones that already exist in WSN,
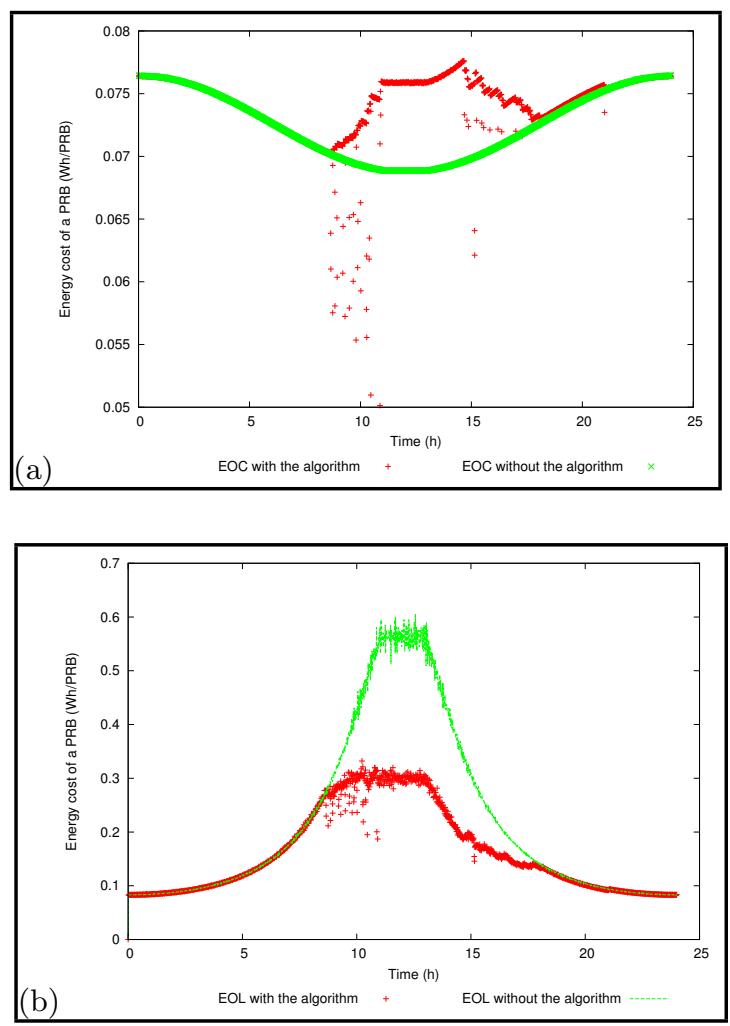

Figure 10: Energy cost of an offered PRB (a) and a used one (b)

coverage and connectivity. In the case of the heterogeneous architecture, only the capacity constraints matter because the coverage is provided by the macro base station. In the settings of LTE mesh networks, one should consider supplementary constraints : coverage on the user link, backhaul connectivity and throughput constraints on the mesh links.

Finally, the difference in the energy models between WSN and cellular networks are not that obvious from the point of view of fixed costs, which, even though they are significantly lower, they do exist in WSN also, but from the perspective of the variation of the power consumption with the load. Wireless sensors are not at all load dependent, while base stations are more or less depending on the type of the eNodeB. As sleep modes are efficient when equipments are little load proportional, it is obvious why they are very popular for WSN and this also means that they are well adapted to micro base stations. But it is rather tempting to think about the case of macro base stations where, even if there is more load proportionality than for the micro eNodeBs, the fixed costs are much more important. Be that as it may, the efficiency of sleep modes is defined in a relative manner (output power fixed power ratio) rather than in an absolute one which would give an advantage to the macro sleep procedures. Besides, switching off a macro eNodeB in a cellular environment is far from being a feasible solution given the characteristics of such a base station (extended range, coverage oriented, equipment complexity etc.).

Our proposition is based on three key points. First, periodically, each eNodeB broadcasts, through X2 interfaces, its current traffic load to its neighborhood. Second, when the load of a micro eNodeB is below a given threshold, it 
checks if its neighborhood has sufficient available capacity to support its remaining load. Note, that this decision is fully local: it means that there is no additional signaling. Third, if there is no sufficient available capacity among its neighborhood, the micro eNodeB stays awake. Otherwise, dedicated control packets are used to notify the neighbors and prepare the forthcoming hand-off. Finally, if every step of the process is successful, the eNodeB switches off. Its neighbors may wake it up afterward if their load rises above another threshold.

The simulation results highlight that, considering a heterogeneous network containing 30 micro eNodeBs, we could expect absolute energy gains of approximately $12 \%$ when implementing sleep modes for the micro eNodeBs. This percentage was obtained for a scenario where the conditions on the thresholds were relatively strong. We also saw that when applying the developed algorithm, the average energy cost of an offered PRB increases by around $2 \%$ while the average energy cost of a used PRB decreases by $24 \%$. This last metric is a good indicator for measuring network energy adaptation to load conditions and therefore energy efficiency of a cellular access network.

Future works will be focused on the control of switch off conditions to avoid a coverage hole on the user side and also to improve the traffic management and the traffic distribution between eNodeBs to save more energy. Investigation on the adaptation of these techniques to LTE mesh networks, on the minimization of the X2 control traffic generated and on the optimal values of the parameters of our propositions are also key features.

\section{REFERENCES}

[1] G. Auer, V. Giannini, I. Godor, P. Skillermark, M. Olsson, M. Ali-Imran, D. Sabella, M. Gonzalez, O. Blume, and A. Fehske. How much energy is needed to run a wireless network? IEEE Transactions on Wireless Communications, Oct. 2011.

[2] J. G. Andrews, H. Claussen, M. Dohler, S. Rangan, and M. C. Reed. Femtocells: Past, Present, and Future. IEEE Journal on Selected Areas in Communications, 30(3):497-508, 2012.

[3] D. Lopez-Perez, I. Guvenc, G. De la Roche, M. Kountouris, T. Quek, and J. Zhang. Enhanced intercell interference coordination challenges in heterogeneous networks. IEEE Wireless Communications, 18(3):22-30, 2011.

[4] M. Marsan, L. Chiaraviglio, D. Ciullo, and M. Meo. Optimal energy savings in cellular access networks. In International Workshop on Green Communications (GreenComm), Dresden, Germany, June 2009.

[5] Greentouch initiative. http://www.greentouch.org/

[6] European Community FP7. Earth Project. http://www.ict-earth.eu/, 2010-2012.

[7] C. Desset, B. Debaillie, V. Giannini, A. Fehske, G. Auer, H. Holtkamp, W. Wajda, D. Sabella, F. Richter, M. Gonzalez, H. Klessig, I. Godor, M. Olsson, M. Imran, A. Ambrosy, and O. Blume. Flexible power modeling of LTE base stations. In IEEE Wireless Communications and Networking Conference (WCNC), pages 2858-2862, Paris, France, Apr. 2012.
[8] A. Fehske, F. Richter, and G. Fettweis. Energy efficiency improvements through micro sites in cellular mobile radio networks. In IEEE GLOBECOM Workshops, Honolulu, Hawaii, USA, Dec. 2009.

[9] C. Rosenberg and J. Ghimire. Resource allocation, transmission coordination and user association policy in heterogeneous cellular networks: a flow based approach. IEEE Transactions on Wireless Communications, 12(1):248-257, Jan. 2013.

[10] A. Ouni, H. Rivano, and F. Valois. Wireless Mesh Networks: Energy - Capacity Tradeoff and Physical Layer Parameters. In IEEE International Symposium on Personal, Indoor and Mobile Radio Communications (PIMRC), Toronto, Canada, Sep. 2011.

[11] A. Capone, I. Filippini, B. Gloss, and U. Barth. Rethinking cellular system architecture for breaking current energy efficiency limits. In IFIP Conference on Sustainable Internet and ICT for Sustainability (SustainIT), Pisa, Italy, Oct. 2012.

[12] L. Chiaraviglio, D. Ciullo, G. Koutitas, L. Tassiulas, and M. Meo. Energy-efficient planning and management of cellular networks. In International Conference on Wireless On-demand Network Systems and Services (WONS), Torino, Italy, Jan. 2012.

[13] C. Khirallah, J. Thompson, and D. Vukobratovic. Energy efficiency of heterogeneous networks in LTE-advanced. In IEEE Wireless Communications and Networking Conference Workshops (WCNC), pages 53-58, Paris, France, Apr. 2012.

[14] L. Chiaraviglio, D. Ciullo, M. Mellia, and M. Meo. Network pruning for energy saving in the Internet. In IEEE INFOCOM 2011 Workshop on Green Communications and Networking, Shanghai, China, Apr. 2011.

[15] D. Tsilimantos, J.-M. Gorce, and E. Altman. Stochastic Analysis of Energy Savings with Sleep Mode in OFDMA Wireless Networks. In IEEE International Conference on Computer Communications (INFOCOM), Torino, Italian, Apr. 2013.

[16] F. Cuomo, A. Cianfrani, M. Polverini, D.Mangione. Flexible power modeling of LTE base stations. Computer Networks, Volume 56, Issue 10, 5 July 2012

[17] C. Huang and Y. Tseng. The coverage problem in a wireless sensor network. Mobile Networks and Applications (MONET), 10(4):519-528, 2005.

[18] C.-F. Huang, Y.-C. Tseng, and H.-L. Wu. Distributed protocols for ensuring both coverage and connectivity of a wireless sensor network. ACM Transactions on Sensor Networks, 3(1):5, 2007.

[19] R. Ramanathan and R. Rosales-Hain. Topology control of multihop wireless networks using transmit power adjustment. In IEEE International Conference on Computer Communications (INFOCOM), Tel-Aviv, Israel, Mar. 2000. 